\title{
LCC from July and August 2019 Lists
}

Compiled by Ann Heinrichs

The new classification numbers listed here reflect the most recent information available at the time of publication. Items in this list were selected from the 2019 list numbers 07 (July 15) and 08 (August 19).

\section{CLASS BF}

BF1622.M35

Occult sciences

Magic (White and Black). Shamanism. Hermetics. Necromancy

By race, ethnic group, or country, A-Z

Malaysians

\section{CLASS BL}

BL820.F56

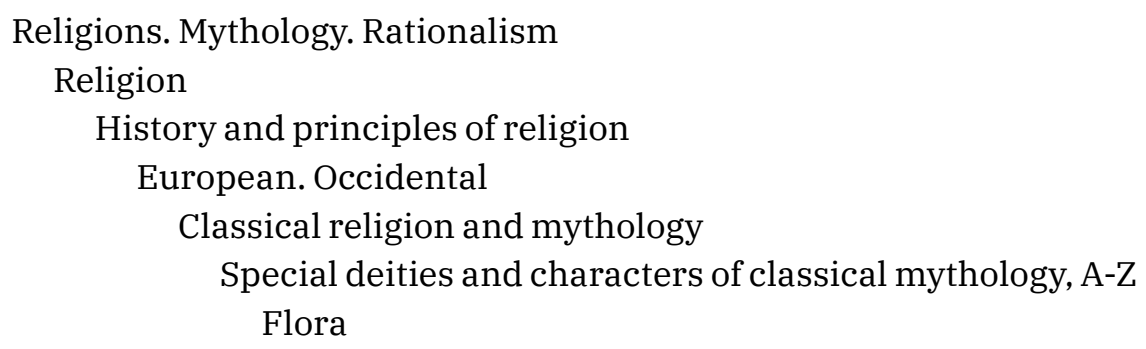

BL1239.82.U43

Umālai Harāuba

\section{CLASS BM}

BM729.P43

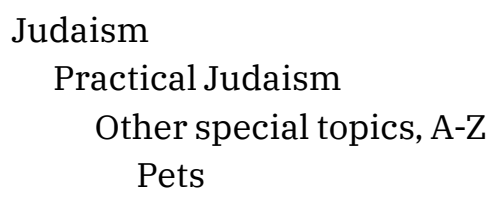


CLASS BP

BP190.5.V625

Islam. Bahai Faith. Theosophy, etc.

Islam

Topics (not otherwise provided for), A-Z

Voluntarism

CLASS BR

BR128.K67

Christianity

Relation of Christianity to other religious and philosophical systems

Special, A-Z

Korean religions

\section{CLASS BS}

BS325.B24

The Bible

General

Texts and versions

Modern texts and versions

Non-European languages

African languages, A-Z

Bakwé TABLE BS5

CLASS BT

BT660.Z3

Doctrinal theology

Mary, Mother of Jesus Christ. Mariology

Miracles. Apparitions. Shrines, sanctuaries, images, processions, etc.

Special

Other, A-Z

Zapopan (Mexico). Our Lady of Zapopan

CLASS KBP

KBP300.M848

شريعة. فقه Islamic law. Sharī ‘ ah. Fiqh

مذاهب Schools of thought. Islamic legal schools. Madhāhib.

Schools and authors affiliated with a particular school

Sunnī schools

Hب̣anafī. Ḥanafīyah. طبقات، الحنفي، الحنفية

Individual authors, A-Z

Mullā, Rā 'id ibn ‘ Abd Allāh ibn Muḥammad. ملا، رائد بن عبد الله بن محمد TABLE K4 
5 THEOLOGY CATALOGING BULLETIN • OCTOBER 2019: VOL. 27, NO 4

\title{
CLASS N
}

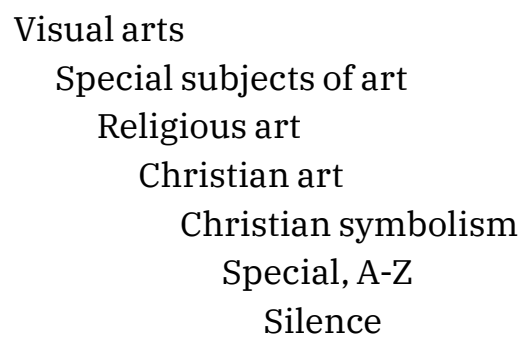

CLASS PN

Drama

Motion pictures

Other special topics, A-Z

PN1995.9.J46 Jews CANCEL

PN1995.9.J46 Jews

Including ethnic and religious subgroups, e.g., Mizrahim

[PN1995.9.M582] Mizrahim see PN1995.9.J46

\section{CLASS PT}

PT415.2.M87

German literature

History of German literature

By period

Modern

21st century

Special topics, A-Z

Muslims

\section{CLASS Z}

Z251.H86

\author{
Book industries and trade \\ Printing \\ Practical printing \\ Type and type founding. Specimen books \\ By language, $\mathrm{A}-\mathrm{Z}$ \\ Hungarian
}

